Resources Flows Asymmetries in Strict Nash Networks with Partner Heterogeneity

Pascal Billand, Christophe Bravard, Sudipta Sarangi

Mars 2011 


\section{GATE Groupe d'Analyse et de Théorie Économique Lyon-St Étienne}

93, chemin des Mouilles 69130 Ecully - France

Tel. +33 (0)4 72866060

Fax $+33(0) 472866090$

6, rue Basse des Rives 42023 Saint-Etienne cedex 02 - France

Tel. +33 (0)4 77421960

Fax. $+33(0) 477421950$

Messagerie électronique / Email : gate@gate.cnrs.fr

Téléchargement / Download : http://www.gate.cnrs.fr - Publications / Working Papers 


\title{
Resources Flows Asymmetries in Strict Nash Networks with Partner Heterogeneity
}

\author{
PASCAL BILLAND ${ }^{a}$, CHRISTOPHE BRAVARD $^{a}$, SUDIPTA SARANGI $^{b}$
}

${ }^{a}$ Université de Lyon, Lyon, F-69003, France ; Université Jean Monnet, Saint-Etienne, F-42000, France ; CNRS, GATE Lyon St Etienne, Saint-Etienne, F-42000, France. email: pascal.billand@univst-etienne.fr, christophe.bravard@univ-st-etienne.fr

${ }^{b}$ Department of Economics, Virginia Tech and Louisiana State University. email: sarangi@lsu.edu

\begin{abstract}
This paper introduces a partner heterogeneity assumption in the one-way flow model of Bala and Goyal (2000, [1]). Our goal consists in the characterization of strict Nash networks with regard to the set of resources obtained by players. We use the notion of condensation network which allows us to divide the population in sets of players who obtain the same resources and we order these sets according to the resources obtained. Accordingly, we can examine the relationship between heterogeneity and asymmetries in networks. We establish that the nature of heterogeneity plays a crucial role on asymmetries observed in equilibrium networks.
\end{abstract}

JEL Classification: C72, D85

Keywords: Nash networks, one-way flow model, condensation networks, chain, inf-semi-lattice.

\section{Introduction}

In many situations agents can obtain resources from others by setting links without the consent of the latter. For example, in the World Wide Web agents can access to the webpage of other agents in order to obtain information without the consent of the webmaster. Likewise peer to peer softwares like Kazaa or Emule generally allows to an agent $i$ to obtain resources from an agent $j$ without the explicit consent of the latter player. Moreover, a distinctive aspect in the previous situations is that 
the benefits or the costs that any agent can obtain from an agent $i$ depends on the characteristics of this agent, that is benefits and costs of links are partner dependent. Thus, some homepages are easier to access on the web as compared to others. Likewise due to the speed connection of agents, ${ }^{1}$ some users are easier to access with peer to peer softwares as compared to others.

A distinctive aspect of previous networks is to allow agents to get access to the resources of each others. In this respect, asymmetries between agents positions, which are a prevalent feature of real networks (See for instance Newman, 2003, [2] or Jackson, 2006, [3]), may play a fundamental role in determining asymmetries between agents with regard to the resources obtained and differences in economic performance.

The goal of this paper consists in analyzing the exchange of resources in the network that results from the formation of links, in situations where benefits and costs of links are partner dependant, and to account for asymmetries with regard to the ressources obtained by agents in these situations. Several notions are useful to conduct the analysis. In particular, we use the notion of condensation network (induced by strict Nash networks) and some notions concerning binary relations.

The notion of condensation networks induced by strict Nash networks allows us to partition agents into groups of agents who obtain resources from the same agents, hence who obtain the same amount of resources in the network, and to display the flows of resources between these groups. In this respect, relative to the notion of strict Nash networks which focus on individual agents and flows of resources between them, the notion of condensation network induced by strict Nash networks is well designed to highlight the asymmetries in resources obtained by groups of agents in networks.

The notions concerning binary relations (chain, inf-semi-lattice) allow us to characterize the properties of the condensation networks induced by strict Nash networks. More precisely, they permit to rank groups of agents with regard to the set of resources obtained. Hence, these notions allow to characterize asymmetries between groups of agents in equilibrium.

In this paper, we focus on two kinds of frameworks. In the first one, values are partner dependent

\footnotetext{
${ }^{1}$ Generally, download speed is higher than upload speed.
} 
while costs are homogeneous. In the second one, both values and costs are partner dependent.

Our main findings are the following.

1. If value is partner dependent while cost of setting link is homogeneous, then there are either 1 or $n$ groups of players (with regard to the values obtained) in strict Nash networks. Moreover, when there are $n$ groups, we can define a chain with regard to the obtained resources relation over the set of players. In other words for any two players $i$ and $j$, either $i$ obtains the resources of $j$, or $j$ obtains the resources of $i$.

2. If both value and cost are partner dependent, then we obtain situations where there exists a specific partial order relation between groups of players with regard to the resources they obtain: a inf-semi-lattice. That is two groups of players always have a greatest lower bound with regard to the resources they obtain.

Our work is related to the non-cooperative literature of network formation initiated by Bala and Goyal (2000, [1]). These authors propose a model, the one-way flow model, where the flow of resources is directed. In such a case, if player $i$ forms an arc with player $j$, then player $i$ obtains resources from $j$ but not vice versa. In their model all players are homogeneous: setting an arc with player $j$ or player $k$ has the same cost for all players and the resources that players obtain from $j$ and $k$ are identical. In this context, Bala and Goyal show that if players' payoffs are increasing in the number of other players accessed and decreasing in the number of arcs formed, then a strict Nash network is either a wheel (a connected network in which each player creates and receives one link), or the empty network. It follows that, in strict equilibria, players are always in a symmetric position with regard to the resources they obtain.

This conclusion is not in line with the most stable empirical findings of networks literature which concern the fact that networks have very asymmetric architectures, and so players do not obtain the same resources.

Recently several papers have examined models of networks formation with heterogeneity. ${ }^{2}$ The pa-

\footnotetext{
${ }^{2}$ Gilles and Johnson (2000, [4]) consider link costs that are increasing in the spatial distance between players while
} 
per that relates most closely to our work is by Galeotti (2006, [10]). The authors studies situations where players are heterogeneous with regard to the costs of linking and the values of accessing other players. More precisely, he introduces two kinds of heterogeneity.

In the first kind, each player $i$ obtains (incurs) the same value (cost) from player $k$. In the sequel we call this framework the player heterogeneity framework. Galeotti shows that if the parameters of the model are such that there is no isolated players in strict equilibrium, that is all players play a role in the network, then there are two cases: either each player obtains the resources of all other players, or the set of players is partitioned into two groups. The members of the first group obtains the resources of all the population while each member of the second group only obtains her own resources. These results leads to the two following conclusions. First, player heterogeneity can produce asymmetries between players with regard to the resources they obtain in strict equilibrium. Second, player heterogeneity leads to very specific situations concerning these asymmetries: either there are no asymmetries between players since each player obtain the resources of all others, or the asymmetries are "very strong" since a group of players obtains the resources of all the population and the other players do not benefit from the network formation.

The second kind of heterogeneity allows cost and value to depend on the identity of both players involved in the relationship, that is the cost and value have two degrees of freedom. In such a case, for any minimal network (networks where there is no superfluous links with regard to the resources obtained by players) there are parameters for which this network is a strict Nash network. In that case all types of asymmetries can arise in strict Nash network with regard to the resources obtained by players. Galeotti (2006, pg.173, [10]) writes "An open question, which is left for further research, McBride (2004, [5]) focuses on value heterogeneity and partial information regarding the network structure. Haller and Sarangi (2005, [6]) propose a model of heterogeneous link reliability and Hojman and Szeidl (2006, [7]) develop a general model of decay where the resources obtained by a player depends on the distance between the players in the network. Galeotti et al. (2006, [8]) introduce heterogeneity in the two-way flow connections model initiated by Bala and Goyal (2000, [1]) by allowing costs and benefits of links to depend on the identity of the player who is forming the links. Finaly, Billand et al. (2010, [9]) examines the impact of the partner heterogeneity on the size of the set of strict Nash networks in the two-way flow model. 
is whether we can say something systematic about the architectural properties of equilibrium networks in partner specific heterogeneous models."

Our work focuses on this issue. It contributes to the literature in the following way. We establish that the dichotomy concerning the asymmetries in strict Nash networks obtained by Galeotti (2006, [10]) results from the player heterogeneity assumption and does not hold anymore when we introduce partner heterogeneity. More precisely, it is not necessary to have two degrees of freedom in cost or in value to obtain intermediate ranges of asymmetries between players with regard to the resources that they obtain in strict Nash networks. To sum up, we know from Bala and Goyal (2000, [1]) that heterogeneity is a necessary condition for strict Nash networks to exhibit asymmetries in resources obtained by players when there is no imperfection concerning the resources flows. We establish that the kind of asymmetries that we obtain depends not only on the number of degree of freedoms of the value and cost parameters but also on the nature of this heterogeneity.

The rest of the paper is organized as follows. Section 2 introduces the model setup and the notions used in the paper. Section 3 presents the results under partner value heterogeneity with homogeneous cost. Section 4 deals with partner value heterogeneity with partner dependent cost. Section 5 concludes.

\section{Model setup}

Networks definitions. We begin by giving the formal definition of a directed network. A network $\boldsymbol{g}$ is an ordered pair of disjoint sets $(V, A)$ such that $A$ is a subset of the set $V^{2}$ of ordered pairs of $V$. The set $V$ is the set of vertices and $A$ is the set of $\operatorname{arcs}$. Let $\mathcal{G}$ be the set of directed network. If $\boldsymbol{g}$ is a directed network, then $V=V(\boldsymbol{g})$ is the vertex set of $\boldsymbol{g}$, and $A=A(\boldsymbol{g})$ is the arc set. An ordered pair $(i, j) \in A(\boldsymbol{g})$ is said to be an arc directed from $i$ to $j$ and is denoted $i j$. If there is an arc from $i \in V(\boldsymbol{g})$ to $j \in V(\boldsymbol{g})$ in $\boldsymbol{g}$, then $i$ is a predecessor of $j$ in $\boldsymbol{g}$. Let $A_{i}(\boldsymbol{g}) \subset A(\boldsymbol{g})$, $A_{i}(\boldsymbol{g})=\{i j \mid j \in N\}$ be the set of arcs directed from $i$ to another vertex. By convention, we assume 
here that there is an arc from each vertex $i$ to herself. For a directed network, $\boldsymbol{g}$, a path $P_{i, j}(\boldsymbol{g})$ in $\boldsymbol{g}$ from (the initial) vertex $i$ to (the terminal) vertex $j$ is an alternating sequence of vertices and arcs: $i_{0}, i_{0} i_{1}, i_{1}, i_{1} i_{2}, \ldots, i_{\ell-1} i_{\ell}, i_{\ell}$ such that $i_{0}=i, i_{\ell}=j$. A cycle is obtained from a path on adding an arc from the terminal vertex to the initial vertex.

We say that $\boldsymbol{g}^{\prime}$ is a sub-network of $\boldsymbol{g}$ if $V\left(\boldsymbol{g}^{\prime}\right) \subset V(\boldsymbol{g})$ and $\boldsymbol{g}^{\prime}$ contains all arcs of $\boldsymbol{g}$ that join two vertices in $V\left(\boldsymbol{g}^{\prime}\right)$. A network is connected if for every pair $(i, j)$ of distinct vertices there is a path from $i$ to $j$. A maximal connected sub-network of $\boldsymbol{g}$ is a component of $\boldsymbol{g}$. The empty network, $\boldsymbol{g}^{e}$, is a network which contains no link between distinct vertices. The network $\boldsymbol{g}$ is a tail star if the set of vertices can be partitioned into two groups $N_{1}=\{1, \ldots, k\}, N_{2}=\{k+1, \ldots, n\}$ such that for all vertices $i \in N_{1}$, we have $i+1 i \in A(\boldsymbol{g})$, and $i j \notin A(\boldsymbol{g})$ otherwise, and for all vertices $i \in N_{2}$, we have $i k \in A(\boldsymbol{g})$, and $i j \notin A(\boldsymbol{g})$ for all $j \neq k$. A line network $\boldsymbol{g}$ is a tail star where $N_{2}=\emptyset$. A center sponsored star is a network where there is a link from a vertex $i_{0}$ to all vertices $j \neq i_{0}$ and there are no other links. Two networks $\boldsymbol{g}$ and $\boldsymbol{g}^{\prime}$ are isomorphic if they have the same number $p$ of vertices and if we can order their vertices respectively $i_{1}, i_{2}, \ldots, i_{p}$ and $j_{1}, j_{2}, \ldots, j_{p}$ so that for any $k$ and $\ell$, arc $i_{k} i_{\ell}$ is in $A(\boldsymbol{g})$ iff $\operatorname{arc} j_{k} j_{\ell}$ is in $A\left(\boldsymbol{g}^{\prime}\right)$.

We now present the notion of condensation network given by Harary, Norman and Cartwright (HNC, 1965, [11]). Let $F_{\boldsymbol{g}}: 2^{V(\boldsymbol{g})} \rightarrow\left\{1, \ldots, 2^{n}\right\}$ be a one to one mapping. $F_{\boldsymbol{g}}$ maps a number to any subset of the set of vertices of $\boldsymbol{g}$. The network $\boldsymbol{g}^{\star}$ is a condensation network induced by the directed network $\boldsymbol{g}$ if the set of vertices satisfies Property 1 and the set of arcs satisfies Property 2.

Property 1. Suppose $X \subseteq V(\boldsymbol{g}) . \quad F_{\boldsymbol{g}}(X) \in V\left(\boldsymbol{g}^{\star}\right)$ iff (i)there is a path from any $i \in X$ to any $j \in X$ in $\boldsymbol{g}$ and (ii)there is no $Y, Y \supset X$ such that there is a path from any $i \in Y$ to any $j \in Y$ in $g$.

Property 2. Suppose $F_{\boldsymbol{g}}(X), F_{\boldsymbol{g}}(Y) \in V\left(\boldsymbol{g}^{\star}\right)$. There is an arc from $F_{\boldsymbol{g}}(X)$ to $F_{\boldsymbol{g}}(Y)$ iff there exist vertices $i \in X$ and $j \in Y$ such that there is an arc from $i$ to $j$ in $A(\boldsymbol{g})$. 
Clearly, these properties induce that the set of vertices of the condensation network $\boldsymbol{g}^{\star}$ is constructed by partitioning the set of vertices $V(\boldsymbol{g})$ with respect to the components of $\boldsymbol{g}$. Moreover, we know from HNC (1965, [11], Theorem 3.2, pg.55) that every vertex is contained in exactly one component and each arc is contained in at most one component. It follows that it is always possible to construct a condensation network from any network. We now give an example of a condensation network $\boldsymbol{g}^{\star}$ induced by a network $\boldsymbol{g}$. Let $N=\{1, \ldots, 6\}$ be the set of vertices of $\boldsymbol{g}$ and the arcs of $\boldsymbol{g}$ are drawn in Figure 2. We observe that vertices 1, 2, 3 are in the same component in $\boldsymbol{g}$, consequently they are associated with the same vertex in $\boldsymbol{g}^{\star}: F_{\boldsymbol{g}}(\{1,2,3\})$. Similarly, vertices 5 and 6 are in the same component in $\boldsymbol{g}$, consequently they are associated with the same vertex in $\boldsymbol{g}^{\star}: F_{\boldsymbol{g}}(\{5,6\})$. The isolated vertex 4 is associated with $F_{\boldsymbol{g}}(\{4\})$ in $\boldsymbol{g}^{\star}$. Lastly, Property 2 concerning the links between vertices in the condensation networks implies that there is an arc from $F_{\boldsymbol{g}}(\{1,2,3\})$ to $F_{\boldsymbol{g}}(\{4\})$.

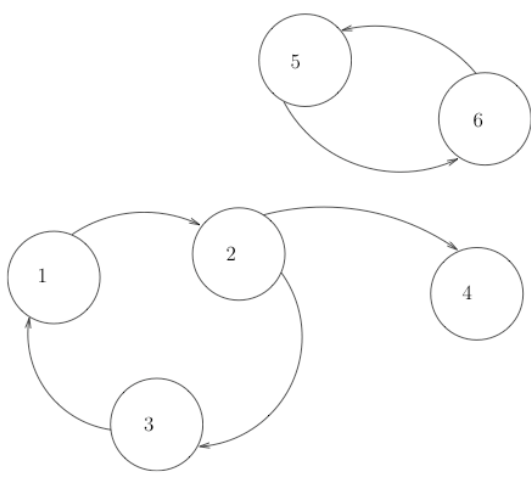

Network $\boldsymbol{g}$

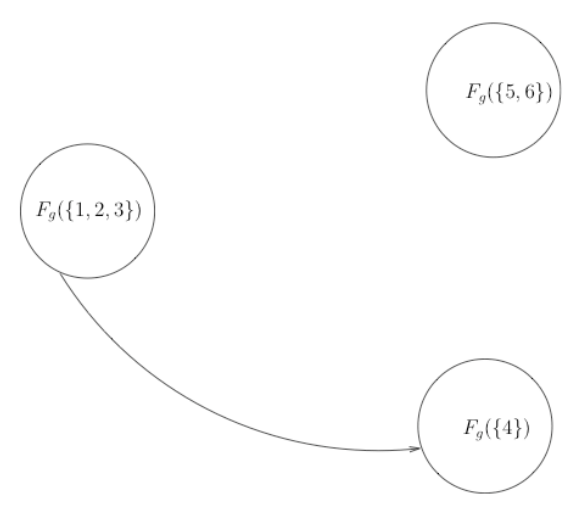

Condensation network $\boldsymbol{g}^{\star}$ induced by $\boldsymbol{g}$

Figure 1: Condensation network

Players and strategies. Due to the notion of condensation network, we will make a distinction between the set of players (or decision makers) and the set of vertices. In particular, we will not assume that the set of players and the set of vertices are necessarily one and the same. Let $N=\{1, \ldots, n\}$ be the set of players. The original network $\boldsymbol{g}$, that is the network formed by the players, is such that $V(\boldsymbol{g})=N$. In $\boldsymbol{g}$ the relations among the players are formally represented 
by the arcs of $\boldsymbol{g}$. Let $G_{i}=\{i j \mid j \in N \backslash\{i\}\}$ be the set of arcs that player $i$ can form with other players. In our context, each player $i \in N$ chooses a strategy which consists in setting arcs: $A_{i}(\boldsymbol{g}) \in 2^{G_{i}}$. If $i j \in A_{i}(\boldsymbol{g})$, then player $i$ has formed an arc with player $j$ in $\boldsymbol{g}$, otherwise player $i$ has not formed an arc with player $j$. In this paper, we only use pure strategies. It is worth noting that the set of arcs between distinct players of network $\boldsymbol{g}$ is $A(\boldsymbol{g})=\bigcup_{i \in N} A_{i}(\boldsymbol{g})$. Given a network $\boldsymbol{g} \in \mathcal{G}$, let $A_{-i}(\boldsymbol{g})=\bigcup_{j \in N \backslash\{i\}} A_{j}(\boldsymbol{g})$ denote the strategy played by all players except $i$. The set of arcs between distinct players of network $\boldsymbol{g}$ can be written as $A(\boldsymbol{g})=A_{i}(\boldsymbol{g}) \cup A_{-i}(\boldsymbol{g})$. Hence if $A_{i}(\boldsymbol{g})=\emptyset$, then $A_{-i}(\boldsymbol{g})=A(\boldsymbol{g})$. It follows that $A_{-i}(\boldsymbol{g})$ is the set of arcs between distinct players of the network obtained from $\boldsymbol{g}$ when all arcs formed by $i$ are removed. To simplify the notations, we write $A_{i}(\boldsymbol{g}) \cup\{i j\}=A_{i}(\boldsymbol{g})+i j$ and $A_{i}(\boldsymbol{g}) \backslash\{i j\}=A_{i}(\boldsymbol{g})-i j$. For consistency, we write $A(\boldsymbol{g}) \cup\{i j\}=A(\boldsymbol{g})+i j$ and $A(\boldsymbol{g}) \backslash\{i j\}=A(\boldsymbol{g})-i j$.

Payoffs. To complete the definition of the normal-form game of network formation, we specify the payoffs. The amount of resources that each player obtains in a network $\boldsymbol{g}$ results from the architecture of $\boldsymbol{g}$. More precisely, an arc $i j$ that player $i$ has formed with player $j$ allows player $i$ to get resources from player $j$ but, since we are in a one-way flow model, this link does not allow $j$ to obtain resources from $i$. Moreover, player $i$ may receive information from other players not only through direct arcs, but also via indirect arcs. To be precise, information flows from player $j$ to player $i$ if $i$ and $j$ are linked by a path from $i$ to $j$ in $\boldsymbol{g}$. Let $N_{i}(A(\boldsymbol{g}))=\{j \in N \mid$ there is a path from $i$ to $j$ in the network $(N, A(\boldsymbol{g}))\}$ be the set of players whom $i$ accesses resources. By construction, we have for each $i, i \in N_{i}(A(\boldsymbol{g}))$. In this paper, we are interested in the partner heterogeneity framework. It follows that each player $j$ allows each player $i$ to obtain $v_{j}>0$ when $j \in N_{i}(\boldsymbol{g})$. Furthermore the formation of an arc with player $j$ implies a cost $c_{j}$ for all players $i \in N \backslash\{j\}$. Since we have postulated that each player $i$ has formed an arc with herself, each player $i$ should obtain her own resources and should incur a cost due to this arc. Since we wish to focus only on the impact of the network on the payoff of players, we do not take into account the effect of the arcs that each player forms with herself on her payoff function. 
Indeed, each player $i$ obtains her own resources even if she forms no arcs, i.e. there is no network. ${ }^{3}$ Finally, to simplify the comparison between the partner heterogeneity framework and the player heterogeneity framework examined by Galeotti, we use the following linear payoff function: ${ }^{4}$

$$
\pi_{i}\left(A_{i}(\boldsymbol{g}), A_{-i}(\boldsymbol{g})\right)=\sum_{j \in N_{i}(A(\boldsymbol{g})) \backslash\{j\}} v_{j}-\sum_{i} c_{j \in A_{i}(\boldsymbol{g})} .
$$

Nash networks and strict Nash networks. The strategy $A_{i}(\boldsymbol{g})$ is said to be a best response of player $i$ against the strategy $A_{-i}(\boldsymbol{g})$ if:

$$
\pi_{i}\left(A_{i}(\boldsymbol{g}), A_{-i}(\boldsymbol{g})\right) \geq \pi_{i}\left(E, A_{-i}(\boldsymbol{g})\right) \text { for all } E \in 2^{G_{i}}
$$

The set of all of player $i$ 's best responses to $A_{-i}(\boldsymbol{g})$ is denoted by $\mathcal{B R}_{i}\left(A_{-i}(\boldsymbol{g})\right)$. A network $\boldsymbol{g}$ is said to be a Nash network if $A_{i}(\boldsymbol{g}) \in \mathcal{B R}_{i}\left(A_{-i}(\boldsymbol{g})\right)$ for each player $i \in N$. We define a strict best response and a strict Nash network by replacing ' $\geq$ ' by ' $>$ '. It is obvious that in a strict Nash network $\boldsymbol{g}$, player $i$ does not form an arc with player $j$ if she obtains the resources of $j$ in the network $\boldsymbol{g}^{\prime}$ with $A\left(\boldsymbol{g}^{\prime}\right)=A(\boldsymbol{g})-i j$. In the sequel, we call this property the basic property of strict Nash networks (BPSN). It is worth noting that BPSN implies that there is at most one link between two vertices of a condensation network induced by a strict Nash network.

Relations: definitions and notations. We define $\succeq_{\boldsymbol{g}}$ as the following binary relation on $V(\boldsymbol{g})$ : $x \succeq_{\boldsymbol{g}} y$ iff there is a path from vertex $x$ to vertex $y$ in $\boldsymbol{g}$. In particular, this definition implies that in the original network $\boldsymbol{g}$, where $V(\boldsymbol{g})=N$, we have $i \succeq_{\boldsymbol{g}} j$ iff $j \in N_{i}(\boldsymbol{g})$. In that case $i \succeq_{\boldsymbol{g}} j$ means that player $i$ obtains resources from player $j$, conversely the dual relation $i \nsucceq_{\boldsymbol{g}} j$ means that

\footnotetext{
${ }^{3}$ A similar assumption is made in Billand et al. (2010, [9]).

${ }^{4}$ The results developped qualitatively carry on when relaxing the linearity assumption of payoff function. However, the payoff function must satisfied the following property: If $\pi_{i}\left(A_{i}(\boldsymbol{g})+i k, A_{-i}(\boldsymbol{g})\right)-\pi_{i}\left(A_{i}(\boldsymbol{g}), A_{-i}(\boldsymbol{g})\right)>0$, and $N_{j}(\boldsymbol{g}) \cap N_{k}(\boldsymbol{g})=\emptyset$, then $\pi_{j}\left(A_{j}(\boldsymbol{g})+j k, A_{-j}(\boldsymbol{g})\right)-\pi_{j}\left(A_{j}(\boldsymbol{g}), A_{-j}(\boldsymbol{g})\right)>0$. In other words, if player $i$ has an incentive to form an arc with player $k$ in $\boldsymbol{g}$ and player $j$ obtains no resources from $k$ in $\boldsymbol{g}$, then player $j$ has an incentive to form an arc with $k$.
} 
player $i$ does not obtain resources from player $j$. In the rest of the paper, the main results concern the properties of $\succeq_{\boldsymbol{g}}$ over $N$ in the original network $\boldsymbol{g}$ and the properties of $\succeq_{\boldsymbol{g}^{\star}}$ over $V\left(\boldsymbol{g}^{\star}\right)$ in the condensation network $\boldsymbol{g}^{\star}$. Let us recall some important classes of binary relations.

Relation $\succeq_{\boldsymbol{g}}$ is reflexive over $V(\boldsymbol{g})$ if $x \succeq_{\boldsymbol{g}} x$ for all $x \in V(\boldsymbol{g})$. Relation $\succeq_{\boldsymbol{g}}$ is total if $x \succeq_{\boldsymbol{g}} y$ or $y \succeq_{\boldsymbol{g}} x$ for all $x, y \in V(\boldsymbol{g})$. Relation $\succeq_{\boldsymbol{g}}$ is symmetric over $V(\boldsymbol{g})$ if $x \succeq_{\boldsymbol{g}} y$ implies $y \succeq_{\boldsymbol{g}} x$ for all $x, y \in V(\boldsymbol{g})$. Relation $\succeq_{\boldsymbol{g}}$ is antisymmetric over $V(\boldsymbol{g})$ if $x \succeq_{\boldsymbol{g}} y$ and $y \succeq_{\boldsymbol{g}} x$ imply $x=y$ for all $x, y \in V(\boldsymbol{g})$. Relation $\succeq_{\boldsymbol{g}}$ over $V(\boldsymbol{g})$ is transitive if $x \succeq_{\boldsymbol{g}} y$ and $y \succeq_{\boldsymbol{g}} z$ imply $x \succeq_{\boldsymbol{g}} z$ for all $x, y$ and $z$ in $V(\boldsymbol{g})$. It is obvious that by construction of $N_{i}(\boldsymbol{g}), \succeq_{\boldsymbol{g}}$ is reflexive and transitive over $N$ in the original network $\boldsymbol{g}$. A relation that is reflexive, symmetric, and transitive is called an equivalence relation. An equivalence relation specifies how to partition a set in subsets called equivalence classes. A partially ordered set $\left(V(\boldsymbol{g}), \succeq_{\boldsymbol{g}}\right)$ is a set $V(\boldsymbol{g})$ on which there is a relation $\succeq_{\boldsymbol{g}}$ that is reflexive, antisymmetric, and transitive. A partially ordered set $\left(V(\boldsymbol{g}), \succeq_{\boldsymbol{g}}\right)$ is a chain if $\succeq_{\boldsymbol{g}}$ is total. A Hasse diagram is a graphical representation of a partially ordered set.

Suppose that $\left(V(\boldsymbol{g}), \succeq_{\boldsymbol{g}}\right)$ is a partially ordered and $X$ is a subset of $V(\boldsymbol{g})$. If $x$ is in $V(\boldsymbol{g})$ and $y \succeq_{\boldsymbol{g}} x$ for each $y$ in $X$, then $x$ is a lower bound for $X$. If the set of lower bounds of $X$ has a greatest element, then this greatest lower bound of $X$ is the infimum of $X$. If two elements $x$ and $y$, of a partially ordered set $V(\boldsymbol{g})$, have a greatest lower bound, it is their meet and it is denoted $x \wedge y$. A partially ordered set $V(\boldsymbol{g})$ that contains the meet of each pair of its elements is a inf-semi-lattice. ${ }^{5}$ It is worth noting that $\boldsymbol{g}^{\star}$, the condensation of a network $\boldsymbol{g}$, allows us to capture the set of players who obtain the same resources. More precisely, all players who belong to $X$ with $F_{\boldsymbol{g}}(X) \in V\left(\boldsymbol{g}^{\star}\right)$ obtain the same resources. In other words, each vertex of $\boldsymbol{g}^{\star}$ is an equivalence class of $\succeq_{\boldsymbol{g}}$ over $N$.

\footnotetext{
${ }^{5}$ See Birkhoff (1993, [12]) for additional information on semi-lattices.
} 


\section{Strict Nash networks with partner value and homoge- neous cost}

In the following, we are interested by ranking the players with regard to the sets of resources that they obtain at equilibrium. Therefore, in this paper, we focus on non-empty strict Nash networks (and their condensation networks) since in the empty network there are no flows of resources between players. We begin with a property which is satisfied by all strict Nash networks in situations where values are partner dependent and costs are homogeneous, that is $c_{j}=c$ for all players $j \in N$.

Proposition 1 Suppose the payoff function of each player $i$ satisfies (1) with $c_{j}=c$ for all $j \in N$. If $\boldsymbol{g}$ is a non-empty strict Nash network, then $\left|V\left(\boldsymbol{g}^{\star}\right)\right| \in\{1, n\}$.

Proof Let $\boldsymbol{g}$ be a non-empty strict Nash network. To introduce a contradiction suppose $\left|V\left(\boldsymbol{g}^{\star}\right)\right| \in$ $\{2, \ldots, n-1\}$. There are two possibilities to obtain such a result given that $|V(\boldsymbol{g})|=n$. Either

there exist $F_{\boldsymbol{g}}(X)$ and $F_{\boldsymbol{g}}(Y)$ in $V\left(\boldsymbol{g}^{\star}\right)$ such that $X, Y \in 2^{N}$ and $|X|,|Y|>1$, or there exists $F_{\boldsymbol{g}}(X)$ in $V\left(\boldsymbol{g}^{\star}\right)$ such that $X \in 2^{N}$ and $n>|X|>1$, and for all $F_{\boldsymbol{g}}(Y)$ in $V\left(\boldsymbol{g}^{\star}\right)$, we have $|Y|=1$. We deal successively with these two possibilities.

1. Suppose $F_{\boldsymbol{g}}(X)$ and $F_{\boldsymbol{g}}(Y)$ in $V\left(\boldsymbol{g}^{\star}\right)$ with $|X|,|Y|>1$. There are two cases: either (i) there is a path from $F_{\boldsymbol{g}}(X)$ (or $\left.F_{\boldsymbol{g}}(Y)\right)$ to $F_{\boldsymbol{g}}(Y)\left(F_{g}(X)\right)$ in $\boldsymbol{g}^{\star}$ or (ii) such a path does not exist.

(i) Suppose wlog that there is a path from $F_{g}(X)$ to $F_{g}(Y)$ with $i \in X$ and $j \in Y$, that is $j \in N_{i}(\boldsymbol{g})$. It follows that there is a path from $k$ to $j$, with $k \in N \backslash Y$. Consequently, there are players $j^{\prime}$ and $k^{\prime}$ in $N$ such that $k^{\prime} j^{\prime} \in A(\boldsymbol{g})$ with $k^{\prime} \in N \backslash Y$ and $j^{\prime} \in Y$. Moreover, since $j^{\prime} \in Y, F_{\boldsymbol{g}}(Y) \in V\left(\boldsymbol{g}^{\star}\right)$ and $|Y|>1$, there is $j^{\prime \prime} \in Y$ such that $j^{\prime \prime} j^{\prime} \in A(\boldsymbol{g})$. Let $\boldsymbol{g}^{\prime}$ be the network where $A\left(\boldsymbol{g}^{\prime}\right)=A(\boldsymbol{g}) \cup\left\{j^{\prime \prime} i\right\} \backslash\left\{j^{\prime \prime} j^{\prime}\right\}$. In $\boldsymbol{g}^{\prime}, j^{\prime \prime}$ incurs the same cost as in $\boldsymbol{g}$ since she forms the same number of arcs, and obtains more resources since by construction $N_{i}\left(\boldsymbol{g}^{\prime}\right) \supset N_{j^{\prime}}(\boldsymbol{g})$. It follows that $\boldsymbol{g}$ is not a strict Nash network, a contradiction.

(ii) Suppose now that there is no path between $F_{\boldsymbol{g}}(X)$ and $F_{\boldsymbol{g}}(Y)$. Since $F_{\boldsymbol{g}}(X) \in V\left(\boldsymbol{g}^{\star}\right)$ (respectively $F_{\boldsymbol{g}}(Y) \in V\left(\boldsymbol{g}^{\star}\right)$ ) and $|X|>1$ (respectively $|Y|>1$ ), there are $i, i^{\prime} \in X$ such that 
$i i^{\prime} \in A(\boldsymbol{g})$ (respectively $j, j^{\prime} \in Y$ such that $j j^{\prime} \in A(\boldsymbol{g})$ ). We show that $\boldsymbol{g}$ is not a strict Nash network. To introduce a contradiction suppose $\boldsymbol{g}$ is a strict Nash network. Assume player $i$ chooses strategy $E_{i}=A_{i}(\boldsymbol{g})-i i^{\prime}+i j^{\prime}$. By using the fact that players $j$ and $j^{\prime}$ belong to $Y$, with $F_{\boldsymbol{g}}(Y) \in V\left(\boldsymbol{g}^{\star}\right)$, it is clear that the marginal payoff obtained by player $i$ is:

$$
\pi_{i}\left(E_{i}, A_{-i}(\boldsymbol{g})\right)-\pi_{i}\left(A_{i}(\boldsymbol{g}), A_{-i}(\boldsymbol{g})\right)=\sum_{\ell \in N_{j}(A(\boldsymbol{g}))} v_{\ell}-\sum_{\substack{\ell \in N_{i}\left(A_{-i}(\boldsymbol{g})+i i^{\prime}\right), \ell \notin N_{i}\left(A(\boldsymbol{g})-i i^{\prime}\right)}} v_{\ell}
$$

which is strictly negative since $\boldsymbol{g}$ is a strict Nash network. Suppose player $j$ chooses strategy $E_{j}=A_{j}(\boldsymbol{g})-j j^{\prime}+j i$. The marginal payoff obtained by player $j$ is:

$$
\pi_{j}\left(E_{j}, A_{-j}(\boldsymbol{g})\right)-\pi_{i}\left(A_{j}(\boldsymbol{g}), A_{-j}(\boldsymbol{g})\right)=\sum_{\ell \in N_{i}(A(\boldsymbol{g}))} v_{\ell}-\sum_{\substack{\ell \in N_{j}\left(A_{-j}(\boldsymbol{g})+j j^{\prime}\right), \ell \notin N_{j}\left(A(\boldsymbol{g})-j j^{\prime}\right)}} v_{\ell} .
$$

which is strictly negative since $\boldsymbol{g}$ is a strict Nash network. Then, we obtain a contradiction since by summing the equations (3) and (4) we obtain:

$$
\sum_{\ell \in N_{j}(A(\boldsymbol{g}))} v_{\ell}-\sum_{\substack{\ell \in N_{i}\left(A_{-i}(\boldsymbol{g})+i i^{\prime}\right), \ell \notin N_{i}\left(A(\boldsymbol{g})-i i^{\prime}\right)}} v_{\ell}+\sum_{\ell \in N_{i}(A(\boldsymbol{g}))} v_{\ell}-\sum_{\substack{\ell \in N_{j}\left(A_{-j}(\boldsymbol{g})+j j^{\prime}\right), \ell \notin N_{j}\left(A(\boldsymbol{g})-j j^{\prime}\right)}} v_{\ell}>0 .
$$

2. Suppose $F_{\boldsymbol{g}}(X) \in V\left(\boldsymbol{g}^{\star}\right)$, with $n>|X|>1$ and let $F_{\boldsymbol{g}}(Y) \in V\left(\boldsymbol{g}^{\star}\right)$, with $|Y|=1$ (we dealt with other cases in the previous point). There are three cases.

(i) There is a path from $F_{\boldsymbol{g}}(Y)$ to $F_{\boldsymbol{g}}(X)$ in $\boldsymbol{g}^{\star}$. In such a case, there are players $i, i^{\prime} \in X$ and $j \in N \backslash X$ such that $i i^{\prime}$ and $j i$ belong to $A(\boldsymbol{g})$. By partner value heterogeneity and cost homogeneity player $j \in N \backslash X$ obtains the same payoff if she replaces her link with $i$ by an arc with $i^{\prime}$. Therefore, $\boldsymbol{g}$ is not a strict Nash network.

(ii) There is a path from $F_{\boldsymbol{g}}(X)$ to $F_{\boldsymbol{g}}(Y)$ in $\boldsymbol{g}^{\star}$. Since $|X|>1$ and $F_{\boldsymbol{g}}(X) \in V\left(\boldsymbol{g}^{\star}\right)$ there are players $i$ and $i^{\prime}$ in $X$ such that $i i^{\prime} \in A(\boldsymbol{g})$. Since there is a path from $F_{\boldsymbol{g}}(X)$ to $F_{\boldsymbol{g}}(Y)$, either player $i$ or player $i^{\prime}$ has formed an arc with a player $j \notin X$ in $\boldsymbol{g}$. Suppose without loss of generality that player $i$ has formed an arc with player $j$ in $\boldsymbol{g}$. Then there is no path, in the 
network associated with $A(\boldsymbol{g})-i j$, from $i^{\prime}$ to $j$ otherwise BSNP is violated. It follows that $\pi_{j}\left(A_{j}(\boldsymbol{g})+j i^{\prime}, A_{-j}(\boldsymbol{g})\right)-\pi_{j}\left(A_{j}(\boldsymbol{g}), A_{-j}(\boldsymbol{g})\right) \geq \pi_{i}\left(A_{i}(\boldsymbol{g}), A_{-i}(\boldsymbol{g})\right)-\pi_{i}\left(A_{i}(\boldsymbol{g})-i i^{\prime}, A_{-i}(\boldsymbol{g})\right)>0$, that is player $j$ obtains the same resources from player $i^{\prime}$ as player $i$ and incurs the same cost when she forms the $\operatorname{arc} j i^{\prime}$. Consequently, $\boldsymbol{g}$ is not a strict Nash network, a contradiction.

(iii) There is no path between $F_{\boldsymbol{g}}(X)$ and $F_{g}(Y)$ in $\boldsymbol{g}^{\star}$. Then we can use the same argument as in the previous point.

If the cost is homogeneous and the values are partner heterogeneous, then the strict Nash condensation networks have either 1 vertex or $n$ vertices. In the former case, all players obtain the same resources.

We now recall two results of directed graph theory useful for Proposition 2.

Theorem 1 (HNC, Theorem 3.7, pg.63, [11]) The following statements are equivalent for any directed network $\boldsymbol{g}$.

1. $\boldsymbol{g}$ is acyclic, that is, has no cycles.

2. $\boldsymbol{g}$ and $\boldsymbol{g}^{\star}$ have the same number of vertices.

3. $\boldsymbol{g}$ and $\boldsymbol{g}^{\star}$ are isomorphic.

Theorem 2 (HNC, Theorem 3.9, pg.65, [11]) The following statements are equivalent for any directed network $\boldsymbol{g}$.

1. There is a path from $i$ to $j$, for any players $i$ and $j$ in $\boldsymbol{g}$.

2. $\boldsymbol{g}^{\star}$ consists of exactly one vertex.

In the following proposition, we will not focus on the condensation network but on the original network $\boldsymbol{g}$. Indeed, in the non-trivial case (that is $\left|V\left(\boldsymbol{g}^{\star}\right)\right|=n$ ) the original network $\boldsymbol{g}$ and its condensation network are isomorphic by Theorem 1 . 
Proposition 2 Suppose the payoff function of each player $i$ satisfies (1) with $c_{j}=c$ for all $j \in N$. Let $\boldsymbol{g}$ be a non-empty strict Nash network. If $\left|V\left(\boldsymbol{g}^{\star}\right)\right|=1$, then $\succeq_{\boldsymbol{g}}$ is an equivalence relation over $N$. If $\left|V\left(\boldsymbol{g}^{\star}\right)\right|=n$, then $\left(N, \succeq_{\boldsymbol{g}}\right)$ is a chain.

Proof Let $\boldsymbol{g}$ be a non empty strict Nash network. Recall that, by construction, $\succeq_{\boldsymbol{g}}$ is reflexive and transitive over $N$.

1. Suppose $\left|V\left(\boldsymbol{g}^{\star}\right)\right|=1$, that is $\boldsymbol{g}^{\star}$ consists of exactly one vertex. We show that $\succeq_{\boldsymbol{g}}$ is symmetric. By Theorem 2 there is a path from $i$ to $j$ and a path from $j$ to $i$, for any players $i$ and $j$ in $\boldsymbol{g}$. It follows that for all $i, j \in N$, we have $i \succeq_{\boldsymbol{g}} j$ and $j \succeq_{\boldsymbol{g}} i$.

2. Suppose $\left|V\left(\boldsymbol{g}^{\star}\right)\right|=n$, we need to show that $\succeq_{\boldsymbol{g}}$ is antisymmetric and total over $N$. Clearly, $\boldsymbol{g}^{\star}$ and $\boldsymbol{g}$ have the same number of vertices: $n$. By Theorem 2, $\boldsymbol{g}$ is acyclic. Consequently, if $i \neq j$, then $i \nsucceq_{\boldsymbol{g}} j$ or $j \succeq_{\boldsymbol{g}} i$, that is $\succeq_{\boldsymbol{g}}$ is antisymmetric over $N$. We now show that $\succeq_{\boldsymbol{g}}$ is total over $N$. In other words, we show that we have either $N_{i}(A(\boldsymbol{g})) \subseteq N_{j}(A(\boldsymbol{g}))$, or $N_{i}(A(\boldsymbol{g})) \supseteq N_{j}(A(\boldsymbol{g}))$ for any players $i, j \in N$.

Firstly, since $\boldsymbol{g}$ (i) is a non empty strict Nash network and (ii) is acyclic, there are players $i, j \in N$ such that $i j \in A(\boldsymbol{g})$ and $N_{j}(A(\boldsymbol{g}))=\{j\}$. If player $i$ has an incentive to form an arc with player $j$ in $\boldsymbol{g}$, then each player $k$ such that $k \notin N_{j}(A(\boldsymbol{g}))$ has an incentive to form an arc with player $j$ in $\boldsymbol{g}$. Indeed, $\pi_{j}\left(A_{j}(\boldsymbol{g})+k j, A_{-j}(\boldsymbol{g})\right)-\pi_{j}\left(A_{j}(\boldsymbol{g}), A_{-j}(\boldsymbol{g})\right)$ $=\pi_{i}\left(A_{i}(\boldsymbol{g}), A_{-i}(\boldsymbol{g})\right)-\pi_{i}\left(A_{i}(\boldsymbol{g})-i j, A_{-i}(\boldsymbol{g})\right)>0$ by partner heterogeneity of value and homogeneity of cost. It follows that $N_{i}(A(\boldsymbol{g})) \cap N_{j}(A(\boldsymbol{g})) \neq \emptyset$ for each $i, j \in N$.

Secondly, we show that there is no player $i$ such that both players $j$ and $k$ have formed an arc with her, that is each player $i$ has at most one predecessor in $\boldsymbol{g}$. To introduce a contradiction suppose that there is a player $i$ with two predecessors $j$ and $k$. Then, player $j$ has an incentive to form an arc with $k$ instead of $i$ since $v_{k}>0$ for all $k \in N$. Since there is a player $j$ such that $j \in N_{i}(A(\boldsymbol{g}))$ for all $i \in N$ and each player $i$ has at most one predecessor, we obtain that $\succeq_{\boldsymbol{g}}$ is total over $N$ by using induction. 
We now show through the following example that there exist some parameters such that $\left(N, \succeq_{\boldsymbol{g}}\right)$ is a chain and $\boldsymbol{g}$ is a strict Nash network.

Example 1 Let $N=\{1,2,3\}, v_{1}=4, v_{2}=v_{3}=1$, and $c=3$. Then the network $\boldsymbol{g}$ such that $A(\boldsymbol{g})=\left\{\begin{array}{lll}2 & 1,3 & 2\end{array}\right\}$ is strict Nash.

Proposition 2 showed that two types of situations arise in non-empty strict Nash networks. In the first one, $\left|V\left(\boldsymbol{g}^{\star}\right)\right|=1$, all players belong to the same equivalence class. In that case, each player obtain the resources of all other players.

In the second one, $\left|V\left(\boldsymbol{g}^{\star}\right)\right|=n,\left(N, \succeq_{\boldsymbol{g}}\right)$ is a chain, that is for each player $i$ and each player $j$, either player $i$ obtains the resources of player $j$ (and player $j$ does not obtain resources of $i$ ) or player $j$ obtains the resources of player $i$ (and player $i$ does not obtain resources of $j$ ). It follows that if cost is homogeneous and value is heterogeneous, then either players are perfectly symmetric, or there is a kind of continuity in the ranking of players, with regard to the resources that players obtained in a strict Nash network. In other words, in the latter case, all players are in an asymmetric position with regard to the resources they access but this asymmetry is "smooth". Finally, we can note by inspecting Example 1 that the player $j$ with the highest value $\left(v_{j} \geq v_{i}\right.$ for all $\left.i \in N\right)$ is also the player who obtains the less resources. It can be shown that the minimal element in $\left(N, \succeq_{g}\right)$ is always the player with the highest value. Hence, in this kind of models, owning the most valuable resources is detrimental to the owner.

We can compare our result with the result obtained by Galeotti in the player heterogeneity framework. Galeotti provides results for cost and value player heterogeneity (Proposition 3.1, pg.169, [10]). These results are preserved when the cost is homogeneous. Clearly, in Galeotti (2006, Proposition 3.1, [10]) the condensation networks induced by non-empty strict Nash networks have $x \in\{1, \ldots, n\}$ vertices. By contrast, in the partner heterogeneity framework the condensation networks induced by non-empty strict Nash networks have either 1 or $n$ vertices. Moreover, in Galeotti, there are some situations such that there is no player who obtains the resources of all other players, that is $\left(N, \succeq_{\boldsymbol{g}}\right)$ has no maximal element. Indeed, in the Galeotti framework, some players can be 
isolated. In such a case, it is not possible to compare some players with others with regard to the set of resources they obtained. In other words, condensation networks induced by non-empty strict Nash network contain groups of players who do not share resources. This result differs from our result since we find that for all players $i$ and $j$, either player $i$ obtains resources of $j$ or $j$ obtains resources of $i$.

We now give the architectures of condensation networks induced by non-empty strict Nash networks. Obviously, if $\left|V\left(\boldsymbol{g}^{\star}\right)\right|=1$, then the condensation network is empty. We now give the architectures of condensation networks induced by non-empty strict Nash networks when $\left|V\left(\boldsymbol{g}^{\star}\right)\right|=n$.

Corollary 1 Suppose the payoff function of each player $i$ satisfies (1) with $c_{j}=c$ for all $j \in N$. If $\left|V\left(\boldsymbol{g}^{\star}\right)\right|=n$, then $\boldsymbol{g}^{\star}$ is a line network.

Proof By Proposition 2, we know that if $\left|V\left(\boldsymbol{g}^{\star}\right)\right|=n$, then $\succeq_{\boldsymbol{g}}$ is a chain over $N$. It follows that $\boldsymbol{g}$ is a line network as the Hasse diagram of a chain. By Theorem 2, $\boldsymbol{g}$ and $\boldsymbol{g}^{\star}$ are isomorphic. Consequently, $\boldsymbol{g}^{\star}$ is a line network.

\section{Strict Nash networks with partner value and cost}

We are now interested in situations with cost and value partner heterogeneity.

Proposition 3 Suppose the payoff function of each player $i$ satisfies (1) and let $\boldsymbol{g}$ be a strict Nash network. If $|X|>1, F_{\boldsymbol{g}}(X) \in V\left(\boldsymbol{g}^{\star}\right)$ and $F_{\boldsymbol{g}}(Y) \in V\left(\boldsymbol{g}^{\star}\right)$, then $|Y|=1$, for all $Y \neq X$.

Proof Let $\boldsymbol{g}$ be a strict Nash network. Suppose there are $|X|>1$ and $|Y|>1$, such that $F_{\boldsymbol{g}}(X) \in V\left(\boldsymbol{g}^{\star}\right)$ and $F_{\boldsymbol{g}}(Y) \in V\left(\boldsymbol{g}^{\star}\right)$. There are two cases: There is a path from $F_{g}(X)$ to $F_{g}(Y)$ (or a path from $F_{g}(Y)$ to $\left.F_{g}(X)\right)$ in $\boldsymbol{g}^{\star}$, or there is no path between $F_{g}(Y)$ to $F_{g}(X)$ in $\boldsymbol{g}^{\star}$. We examine these two cases successively.

1. Suppose wlog that there is a path from $F_{g}(X)$ to $F_{g}(Y)$ in $\boldsymbol{g}^{\star}$. Let $i \in X$ and $j \in Y$. We have $j \in N_{i}(\boldsymbol{g})$. It follows that there exists a player $k \in N \backslash Y$, such that there is a path from $k$ 
to $j$. Consequently, there are players $j^{\prime}$ and $k^{\prime}$ in $\boldsymbol{g}$ such that $k^{\prime} j^{\prime} \in A(\boldsymbol{g})$ with $k^{\prime} \in N \backslash Y$ and $j^{\prime} \in Y$. Moreover, since $j^{\prime} \in Y, F_{\boldsymbol{g}}(Y) \in V\left(\boldsymbol{g}^{\star}\right)$, and $|Y|>1$, there is $j^{\prime \prime} \in Y$ such that $j^{\prime \prime} j^{\prime} \in A(\boldsymbol{g})$. Likewise, since $F_{\boldsymbol{g}}(X) \in V\left(\boldsymbol{g}^{\star}\right)$, and $|X|>1$, there exist players $i$ and $i^{\prime}$ in $X$ such that $i i^{\prime} \in A(\boldsymbol{g})$. Let $\boldsymbol{g}^{\prime}$ be the network where $A\left(\boldsymbol{g}^{\prime}\right)=A(\boldsymbol{g}) \cup\left\{j^{\prime \prime} i^{\prime}\right\} \backslash\left\{j^{\prime \prime} j^{\prime}\right\}$. We have:

$$
\pi_{j^{\prime \prime}}\left(A_{j^{\prime \prime}}\left(\boldsymbol{g}^{\prime}\right), A_{-j^{\prime \prime}}\left(\boldsymbol{g}^{\prime}\right)\right)-\pi_{j^{\prime \prime}}\left(A_{j^{\prime \prime}}(\boldsymbol{g}), A_{-j^{\prime \prime}}(\boldsymbol{g})\right)=c_{j^{\prime}}+\sum_{\ell \in N_{i}(A(\boldsymbol{g})) \backslash N_{j^{\prime}}(A(\boldsymbol{g}))} v_{\ell}-c_{i^{\prime}}
$$

which is strictly negative since $\boldsymbol{g}$ is strict Nash. Let $\boldsymbol{g}^{\prime \prime}$ be the network where $A\left(\boldsymbol{g}^{\prime \prime}\right)=$ $A(\boldsymbol{g}) \cup\left\{\begin{array}{ll}i & j^{\prime}\end{array} \backslash\left\{\begin{array}{ll}i & i^{\prime}\end{array}\right\}\right.$. We have:

$$
\pi_{i}\left(A_{i}\left(\boldsymbol{g}^{\prime \prime}\right), A_{i}\left(\boldsymbol{g}^{\prime \prime}\right)\right)-\pi_{i}\left(A_{i}(\boldsymbol{g}), A_{-i}(\boldsymbol{g})\right)=c_{i^{\prime}}-\sum_{\substack{\ell \in N_{i}(A(\boldsymbol{g})) \\ \backslash N_{i}\left(A\left(\boldsymbol{g}^{\prime \prime}\right)\right)}} v_{\ell}-c_{j^{\prime}}
$$

which is strictly negative since $\boldsymbol{g}$ is strict Nash. These conditions cannot be simultaneously satisfied since $N_{j^{\prime}}(A(\boldsymbol{g})) \subseteq N_{i}\left(A\left(\boldsymbol{g}^{\prime \prime}\right)\right)$. It follows that $\boldsymbol{g}$ is not a strict Nash network.

2. Secondly, suppose that there is no path between $F_{g}(X)$ and $F_{g}(Y)$ in $\boldsymbol{g}^{\star}$. Since $|X|,|Y|>1$ and $F_{g}(X), F_{g}(Y) \in V\left(\boldsymbol{g}^{\star}\right)$, there are players $i, i^{\prime} \in X$ and $j, j^{\prime} \in Y$ such that $i i^{\prime} \in A(\boldsymbol{g})$ and $j j^{\prime} \in A(\boldsymbol{g})$. Suppose player $i$ replaces the link $i i^{\prime}$ by the link $i j^{\prime}$. By using the fact that players $j$ and $j^{\prime}$ are in the same component, the marginal payoff obtained by player $i$ is:

$$
\sum_{\ell \in N_{j}(A(\boldsymbol{g}))} v_{\ell}-\sum_{\ell \in N_{i}\left(A_{-i}(\boldsymbol{g})+i i^{\prime}\right),} v_{\ell}-c_{j^{\prime}}+c_{i^{\prime}}
$$

which is strictly negative since $\boldsymbol{g}$ is strict Nash network.

Suppose player $j$ replaces the link $j j^{\prime}$ by the link $j i^{\prime}$. The marginal payoff obtained by player $j$ is:

$$
\sum_{\ell \in N_{i}(A(\boldsymbol{g}))} v_{\ell}-\sum_{\substack{\ell \in N_{j}\left(A_{-j}(\boldsymbol{g})+j j^{\prime}\right), \ell \notin N_{j}\left(A(\boldsymbol{g})-j j^{\prime}\right)}} v_{\ell}-c_{i^{\prime}}+c_{j^{\prime}}
$$


which is strictly negative since $\boldsymbol{g}$ is strict Nash network.

We obtain a contradiction since by summing Equations 5 and 6, we have:

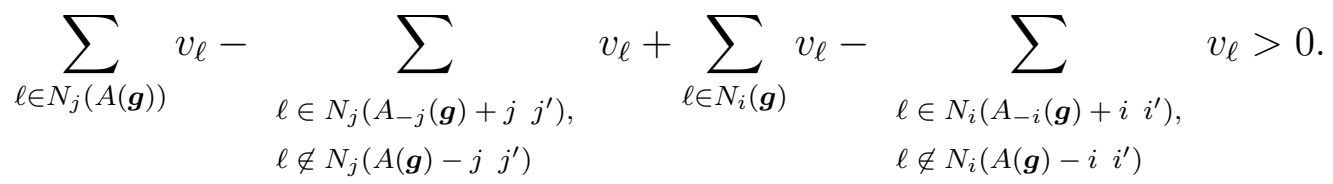

Proposition 3 highlights the fact that there is at most one equivalence class with several players with regard to the set of resources that players obtain.

Let us provide a useful result of graph theory for Proposition 4.

Theorem 3 (HNC, Theorem 3.6, pg.63, [11]) The condensation $\boldsymbol{g}^{\star}$ of any directed network $\boldsymbol{g}$ is acyclic.

Let $M\left(\boldsymbol{g}^{\star}\right)=\left\{F_{\boldsymbol{g}}(X) \in V\left(\boldsymbol{g}^{\star}\right) \mid\right.$ there is no $F_{\boldsymbol{g}}(Y) \in V\left(\boldsymbol{g}^{\star}\right)$ such that $\left.F_{\boldsymbol{g}}(Y) \succeq_{\boldsymbol{g}^{\star}} F g(X)\right\}$ be the set of greatest elements of $\left(V\left(\boldsymbol{g}^{\star}\right), \succeq \boldsymbol{g}^{\star}\right)$. We use two lemmas given in Appendix to establish Proposition 4.

Proposition 4 Suppose the payoff function of each player $i$ satisfies (1) and let $\boldsymbol{g}$ be a non-empty strict Nash network. Then,

1. $\left(V\left(\boldsymbol{g}^{\star}\right), \succeq_{\boldsymbol{g}^{\star}}\right)$ is a inf-semi-lattice. Moreover, If $F_{\boldsymbol{g}}(X) \in V\left(\boldsymbol{g}^{\star}\right)$ is not the minimal element of $\left(V\left(\boldsymbol{g}^{\star}\right), \succeq_{\boldsymbol{g}^{\star}}\right)$, then $|X|=1$.

2. $\left(V\left(\boldsymbol{g}^{\star}\right) \backslash M\left(\boldsymbol{g}^{\star}\right), \succeq_{\boldsymbol{g}^{\star}}\right)$ is a chain and for all $F_{\boldsymbol{g}}(X), F_{\boldsymbol{g}}(Y) \in M\left(\boldsymbol{g}^{\star}\right)$, we have $F_{\boldsymbol{g}}(X) \wedge F_{\boldsymbol{g}}(Y)=$ $F_{\boldsymbol{g}}(Z)$ where $F_{\boldsymbol{g}}(Z)$ is the maximal element of $\left(V\left(\boldsymbol{g}^{\star}\right) \backslash M\left(\boldsymbol{g}^{\star}\right), \succeq_{\boldsymbol{g}^{\star}}\right)$.

Proof We prove successively the two parts of the Proposition. To avoid trivialities, we assume that $\left|V\left(\boldsymbol{g}^{\star}\right)\right| \geq 3$.

1. Firstly, we show that $\succeq_{\boldsymbol{g}^{\star}}$ is a partial order over $\boldsymbol{g}^{\star}$. We know that $\succeq_{\boldsymbol{g}^{\star}}$ is transitive and reflexive over $V\left(\boldsymbol{g}^{\star}\right)$. We need to show that $\succeq_{\boldsymbol{g}^{\star}}$ is antisymmetric over $V\left(\boldsymbol{g}^{\star}\right)$. By Theorem 
3, we know that $\boldsymbol{g}^{\star}$ is acyclic. Consequently, if $i \neq j$, then $i \nsucceq_{\boldsymbol{g}} j$ or $j \nsucceq_{\boldsymbol{g}} i$, that is $\succeq_{\boldsymbol{g}}$ is antisymmetric over $V\left(\boldsymbol{g}^{\star}\right)$.

Secondly, we need to show that for all $F_{\boldsymbol{g}}(X), F_{\boldsymbol{g}}(Y)$ in $V\left(\boldsymbol{g}^{\star}\right)$, there is $F_{\boldsymbol{g}}(Z)$ such that $F_{\boldsymbol{g}}(X) \succeq_{\boldsymbol{g}^{\star}} F_{\boldsymbol{g}}(Z)$ and $F_{\boldsymbol{g}}(Y) \succeq_{\boldsymbol{g}^{\star}} F_{\boldsymbol{g}}(Z)$. We know by Lemma 1 that there is a player $i_{0}$ such that $j \succeq_{\boldsymbol{g}} i_{0}$, for all $j \in N$. Suppose that $i_{0} \in Z$, then we have for all $F_{\boldsymbol{g}}(X) \in V\left(\boldsymbol{g}^{\star}\right)$ $F_{\boldsymbol{g}}(X) \succeq_{\boldsymbol{g}^{\star}} F_{\boldsymbol{g}}(Z)$ by construction of $\boldsymbol{g}^{\star}$. It follows that $\left(V\left(\boldsymbol{g}^{\star}\right), \succeq_{\boldsymbol{g}^{\star}}\right)$ is a inf-semi-lattice.

We now show that if $F_{\boldsymbol{g}}(X) \in V\left(\boldsymbol{g}^{\star}\right)$ is not the minimal element of $\left(V\left(\boldsymbol{g}^{\star}\right), \succeq_{\boldsymbol{g}^{\star}}\right)$, then $|X|=1$. To introduce a contradiction, suppose that $F_{\boldsymbol{g}}(X) \in V\left(\boldsymbol{g}^{\star}\right)$ is not the lower bound over $V\left(\boldsymbol{g}^{\star}\right)$ and $|X|>1$. Since $F_{\boldsymbol{g}}(X)$ is not the minimal element and $\left(V\left(\boldsymbol{g}^{\star}\right), \succeq_{\boldsymbol{g}^{\star}}\right)$ is a inf-semi-lattice, there is a vertex $F_{\boldsymbol{g}}(Y) \in V\left(\boldsymbol{g}^{\star}\right)$ such that $F_{\boldsymbol{g}}(X) F_{\boldsymbol{g}}(Y) \in A\left(\boldsymbol{g}^{\star}\right)$. Since $|X|>1$ there are players $i, i^{\prime} \in X$ such that $i i^{\prime} \in A(\boldsymbol{g})$ and since $F_{\boldsymbol{g}}(X) \succeq_{\boldsymbol{g}^{\star}} F_{\boldsymbol{g}}(Y)$, there are players $i^{\prime \prime} \in X$ and $j \in Y$ such that $i^{\prime \prime} j \in A(\boldsymbol{g})$. Without loss of generality, we assume that $i^{\prime \prime}=i$. By construction of $\boldsymbol{g}^{\star}, j$ does not obtain the resources of players in $X$. If player $j$ forms an arc with $i^{\prime}$, then she obtains resources at least equal to the resources obtained by $i$ due to the arc $i i^{\prime}$ and incurs the same cost. It follows that $j$ has an incentive to form the arc $j i^{\prime}$ since $i$ has an incentive to form an arc with $i^{\prime}$. Therefore, $j$ does not play a strict best response in $\boldsymbol{g}$ and $\boldsymbol{g}$ is not a strict Nash network, a contradiction.

2. We now consider the second part of the Proposition.

Firstly, we establish that $\left(V\left(\boldsymbol{g}^{\star}\right) \backslash M\left(\boldsymbol{g}^{\star}\right), \succeq_{\boldsymbol{g}^{\star}}\right)$ is a chain. We know that $\left(V\left(\boldsymbol{g}^{\star}\right), \succeq_{\boldsymbol{g}^{\star}}\right)$ is a partial order. We need to show that $\succeq_{\boldsymbol{g}^{\star}}$ is total over $V\left(\boldsymbol{g}^{\star}\right) \backslash M\left(\boldsymbol{g}^{\star}\right)$, that is either $F_{\boldsymbol{g}}(X) \succeq_{\boldsymbol{g}^{\star}} F_{\boldsymbol{g}}(Y)$ or $F_{\boldsymbol{g}}(X) \succeq_{\boldsymbol{g}^{\star}} F_{\boldsymbol{g}}(Y)$, for all $F_{\boldsymbol{g}}(X), F_{\boldsymbol{g}}(Y) \in V\left(\boldsymbol{g}^{\star}\right) \backslash M\left(\boldsymbol{g}^{\star}\right)$. To introduce a contradiction suppose that $F_{\boldsymbol{g}}(X) \nsucceq_{\boldsymbol{g}^{\star}} F_{\boldsymbol{g}}(Y)$ and $F_{\boldsymbol{g}}(Y) \nsucceq_{\boldsymbol{g}^{\star}} F_{\boldsymbol{g}}(X)$. Since, $\left(V\left(\boldsymbol{g}^{\star}\right), \succeq_{\boldsymbol{g}^{\star}}\right)$ is a inf-semi-lattice and there exist $F_{\boldsymbol{g}}(X), F_{\boldsymbol{g}}(Y) \in V\left(\boldsymbol{g}^{\star}\right) \backslash M\left(\boldsymbol{g}^{\star}\right)$ such that $F_{\boldsymbol{g}}(X) \nsucceq_{\boldsymbol{g}^{\star}} F_{\boldsymbol{g}}(Y)$ and $F_{\boldsymbol{g}}(Y) \nsucceq_{\boldsymbol{g}^{\star}} F_{\boldsymbol{g}}(X)$, there is a vertex in $\boldsymbol{g}^{\star}$ which has two predecessors. Let $K\left(\boldsymbol{g}^{\star}\right)=\left\{F_{\boldsymbol{g}}(X) \in V\left(\boldsymbol{g}^{\star}\right) \backslash M\left(\boldsymbol{g}^{\star}\right) \mid\right.$ there exist $F_{\boldsymbol{g}}(Y), F_{\boldsymbol{g}}(Z) \in V\left(\boldsymbol{g}^{\star}\right) \backslash M\left(\boldsymbol{g}^{\star}\right)$ : $\left.F_{\boldsymbol{g}}(Y) F_{\boldsymbol{g}}(X), F_{\boldsymbol{g}}(Z) F_{\boldsymbol{g}}(X) \in A\left(\boldsymbol{g}^{\star}\right)\right\}$ be the set of vertices in $V\left(\boldsymbol{g}^{\star}\right) \backslash M\left(\boldsymbol{g}^{\star}\right)$ which have two predecessors in $\boldsymbol{g}^{\star}$. Since $K\left(\boldsymbol{g}^{\star}\right)$ is finite and $\left(V\left(\boldsymbol{g}^{\star}\right), \succeq \boldsymbol{g}^{\star}\right)$ is a inf-semi-lattice, $\left(K\left(\boldsymbol{g}^{\star}\right), \succeq_{\boldsymbol{g}^{\star}}\right)$ 
admits a minimal element, say $F_{\boldsymbol{g}}\left(X_{0}\right)$. Let $F_{\boldsymbol{g}}\left(Y_{0}\right)$ and $F_{\boldsymbol{g}}\left(Z_{0}\right)$ be two vertices such that $F_{\boldsymbol{g}}\left(Y_{0}\right) F_{\boldsymbol{g}}\left(X_{0}\right), F_{\boldsymbol{g}}\left(Z_{0}\right) F_{\boldsymbol{g}}\left(X_{0}\right) \in A\left(\boldsymbol{g}^{\star}\right) . F_{\boldsymbol{g}}\left(Y_{0}\right)$ and $F_{\boldsymbol{g}}\left(Z_{0}\right)$ cannot have an arc in $\boldsymbol{g}^{\star}$ with a vertex $F_{\boldsymbol{g}}(X) \neq F_{\boldsymbol{g}}\left(X_{0}\right)$ otherwise either $K\left(\boldsymbol{g}^{\star}\right)$ is not a finite set which admits $F_{\boldsymbol{g}}\left(X_{0}\right)$ as minimal element, or $\left(V\left(\boldsymbol{g}^{\star}\right), \succeq_{\boldsymbol{g}^{\star}}\right)$ is not a inf-semi-lattice. Moreover, since $F_{\boldsymbol{g}}\left(Y_{0}\right) \in V\left(\boldsymbol{g}^{\star}\right) \backslash M\left(\boldsymbol{g}^{\star}\right)$ there exists a player $F_{\boldsymbol{g}}\left(Y_{1}\right) \in V\left(\boldsymbol{g}^{\star}\right)$ such that $F_{\boldsymbol{g}}\left(Y_{1}\right) F_{\boldsymbol{g}}\left(Y_{0}\right) \in A\left(\boldsymbol{g}^{\star}\right)$. Besides, we know by Proposition 4.1 that $\left|Z_{0}\right|=\left|Y_{0}\right|=\left|Y_{1}\right|=1$. Consequently, the assumptions of Lemma 2 are satisfied. It follows that $\boldsymbol{g}$ is not a strict Nash network, a contradiction.

Secondly, we establish that for all $F_{\boldsymbol{g}}(X), F_{\boldsymbol{g}}(Y) \in M\left(\boldsymbol{g}^{\star}\right)$, we have $F_{\boldsymbol{g}}(X) \wedge F_{\boldsymbol{g}}(Y)=F_{\boldsymbol{g}}(Z)$ where $F_{\boldsymbol{g}}(Z)$ is the maximal element of $\left(V\left(\boldsymbol{g}^{\star}\right) \backslash M\left(\boldsymbol{g}^{\star}\right), \succeq \boldsymbol{g}^{\star}\right)$. We know that this maximal element exists. Indeed, $\left(V\left(\boldsymbol{g}^{\star}\right) \backslash M\left(\boldsymbol{g}^{\star}\right), \succeq_{\boldsymbol{g}^{\star}}\right)$ is a chain, so it contains a maximal element. Moreover, since there is a player $i \in N$ such that $j \succeq_{\boldsymbol{g}} i$ for all $j \in N$, for each $F_{\boldsymbol{g}}(X) \in M\left(\boldsymbol{g}^{\star}\right)$ there exists $F_{\boldsymbol{g}}\left(X^{\prime}\right) \in V\left(\boldsymbol{g}^{\star}\right) \backslash M\left(\boldsymbol{g}^{\star}\right)$ such that $F_{\boldsymbol{g}}(X) F_{\boldsymbol{g}}\left(X^{\prime}\right) \in A\left(\boldsymbol{g}^{\star}\right)$. We now show that $F_{\boldsymbol{g}}(X), F_{\boldsymbol{g}}(Y) \in M\left(\boldsymbol{g}^{\star}\right)$ have formed an arc with the same vertex $F_{\boldsymbol{g}}\left(Z^{\prime}\right) \in V\left(\boldsymbol{g}^{\star}\right) \backslash M\left(\boldsymbol{g}^{\star}\right)$. To introduce a contradiction, suppose that $F_{\boldsymbol{g}}(X), F_{\boldsymbol{g}}(Y) \in M\left(\boldsymbol{g}^{\star}\right)$ are such that $F_{\boldsymbol{g}}(X) F_{\boldsymbol{g}}\left(X^{\prime}\right) \in$ $A\left(\boldsymbol{g}^{\star}\right)$ and $F_{\boldsymbol{g}}(Y) F_{\boldsymbol{g}}\left(Y^{\prime}\right) \in A\left(\boldsymbol{g}^{\star}\right)$ with $X^{\prime} \neq Y^{\prime}$. Clearly $F_{\boldsymbol{g}}\left(X^{\prime}\right), F_{\boldsymbol{g}}\left(Y^{\prime}\right) \in V\left(\boldsymbol{g}^{\star}\right) \backslash M\left(\boldsymbol{g}^{\star}\right)$. Moreover, since $\left(V\left(\boldsymbol{g}^{\star}\right) \backslash M\left(\boldsymbol{g}^{\star}\right), \succeq_{\boldsymbol{g}^{\star}}\right)$ is a chain we can assume wlog that $F_{\boldsymbol{g}}\left(X^{\prime}\right) \succeq_{\boldsymbol{g}^{\star}} F_{\boldsymbol{g}}\left(Y^{\prime}\right)$. Hence there is a vertex $F_{\boldsymbol{g}}\left(Y^{\prime \prime}\right) \in\left(V\left(\boldsymbol{g}^{\star}\right) \backslash M\left(\boldsymbol{g}^{\star}\right)\right) \backslash\left\{F_{\boldsymbol{g}}(Y)\right\}$ such that $F_{\boldsymbol{g}}\left(Y^{\prime \prime}\right) F_{\boldsymbol{g}}(Y) \in A\left(\boldsymbol{g}^{\star}\right)$. We know by Proposition 4.1 that $\left|Y^{\prime \prime}\right|=|Y|=1$. By BPSN player $i_{Y} \in Y$ do not form a link with a player $j \notin Y^{\prime}$. To sum up, there is a player $i_{Y^{\prime \prime}} \in Y^{\prime \prime}$ who forms a unique link with a player $i_{Y^{\prime}} \in Y^{\prime}$ and a player $i_{Y^{\prime \prime}} \in Y^{\prime \prime}$ who forms a unique link with a player $i_{Y^{\prime}}^{\prime} \in Y$. By cost heterogeneity and strict Nash property of $\boldsymbol{g}, i_{Y^{\prime}}^{\prime}=i_{Y^{\prime}}$. Finally, since $F_{\boldsymbol{g}}\left(Y^{\prime \prime}\right)$ belongs to a chain there is a vertex $F_{\boldsymbol{g}}\left(Y^{\prime \prime \prime}\right)$ such that $F_{\boldsymbol{g}}\left(Y^{\prime \prime \prime}\right) F_{\boldsymbol{g}}\left(Y^{\prime \prime}\right) \in A\left(\boldsymbol{g}^{\star}\right)$, with $\left|Y^{\prime \prime \prime}\right|=1$. It follows that there are four players $i_{Y} \in Y, i_{Y^{\prime}} \in Y^{\prime}, i_{Y^{\prime \prime}} \in Y^{\prime \prime}$ and $i_{Y^{\prime \prime \prime}} \in Y^{\prime \prime \prime}$ who satisfy assumptions of Lemma 2. We conclude that $\boldsymbol{g}$ is not a strict Nash network, a contradiction.

The first part of Proposition 4 contains two information. Firstly, the condensation network induced a strict Nash network is not always a lattice. In other words, there exist situations such 
that there are several players whom resources are not accessed by others. However, since the condensation network induced a strict Nash network is inf-semi-lattice, there is a player, say $i_{0}$, such that all players $j \in N$ obtain the resources of $i_{0}$ in a non-empty strict Nash network. Secondly, if several players belong to the same equivalence class with regard to the set of resources obtained, then they do not obtain resources from players who do not belong to this class.

The second part of Proposition 4 highlights the existence of two sets of players. Players who belong to the first set, $M\left(\boldsymbol{g}^{\star}\right)$, are such that they obtain the resources of all players who do not belong to this set, but no player in the population access to the resources owned by these players. Players who belong to the second set, $N \backslash M\left(\boldsymbol{g}^{\star}\right)$, are such that each of them has a predecessor. Hence, there is a hierarchy between players who belongs to this set.

Corollary $\mathbf{2}$ Suppose the payoff function of each player $i$ satisfies (1) and let $\boldsymbol{g}$ be a non-empty strict Nash network. Then, $\boldsymbol{g}^{\star}$ is a tail star.

Proof Let $\boldsymbol{g}$ be a non-empty strict Nash network. Given Proposition 4 the Hasse diagram of $\boldsymbol{g}^{\star}$ is a tail star.

Obviously, if $\boldsymbol{g}^{\star}$ contains 1 vertex, then $\boldsymbol{g}^{\star}$ is empty.

Let us now show through an example that there are situations such that the condensation network induced by a strict Nash network is a tail star.

Example 2 Suppose $N=\{1,2,3,4\}$. Let $v_{1}=v_{2}=1, v_{3}=v_{4}=4, c_{1}=c_{2}=7, c_{3}=1$, and $c_{4}=2$. Straightforward computations show that the network $\boldsymbol{g}$, drawn in Figure 4 , is a strict Nash network. Clearly, $\boldsymbol{g}^{\star}$, drawn in Figure 4, is the condensation network induced by $\boldsymbol{g}$.

We now compare the result given in Corollary ?? with the result obtained in the player heterogeneity framework by Galeotti (2006, Proposition 3.1, pg.169, [10]). Let us recall that in the player heterogeneity framework, condensation networks induced by non-empty strict Nash networks are either empty, or center sponsored stars. Let us deal with situations where condensation networks induced by non-empty strict Nash networks are center sponsored stars. In a center sponsored star, $\boldsymbol{g}^{\star}$ there is one equivalence class which obtains the resources of all other equivalence classes while 


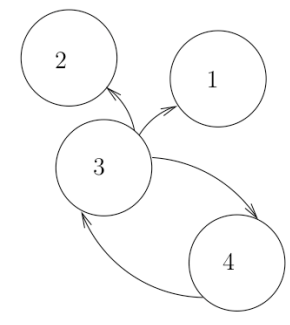

Network $\boldsymbol{g}$

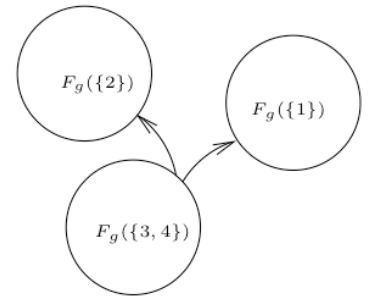

Condensation network $\boldsymbol{g}^{\star}$

Figure 2: Condensation network

the latter obtain no resources from other equivalence classes. In other words, if the center sponsored stars contains $x$ vertices, then there is a vertex, say $F_{\boldsymbol{g}}\left(X_{0}\right)$, which obtains the resources of all other $x-1$ vertices. Therefore, players in $X_{0}$ obtain resources of all other players. Moreover, other vertices cannot be compared between them with regard to the relation $\succeq_{g^{\star}}$. Hence in the player heterogeneity model there is a dichotomy with regard to the resources obtained by players. Indeed, players in one equivalence class obtain resources from all other players while other players obtain only their own resources. By contrast, in the partner heterogeneity model, situations with no dichotomy can arise when the condensation of a non-empty strict Nash network is non empty. Indeed, when the condensation network is a tail star, some players obtain resources from a subset of the population.

Finally, the cost does not play the same role in the player heterogeneity framework and in the partner heterogeneity framework. In the former framework, the results obtain when the cost is homogeneous and the results obtain when the cost is player heterogeneous are qualitatively equivalent. By contrast, in our framework the set of networks which are candidates as strict Nash is larger when the cost is partner heterogeneous than when the cost is homogeneous. Formally, a nonempty condensation network induced by a strict Nash network is a lattice, that is a inf-semi-lattice where each couple of vertices has a least upper bound, ${ }^{6}$ when the cost is homogenous while it is a

\footnotetext{
${ }^{6} \mathrm{~A}$ chain is a lattice.
} 
inf-semi-lattice when the cost is partner heterogeneous.

\section{Conclusion}

The most stable empirical finding concerning the structural properties that networks exhibit in reality is that networks have very asymmetric architectures (concerning the asymmetric properties of the World Wide Web network see for instance Barabási, Albert and Jeong, 2000 [13]). Hence, the study of the determinants of asymmetries is crucial. In this paper, we establish that the nature of the heterogeneity plays an important role in the asymmetries observed in the equilibrium networks. To obtain such a result, we use some tools which allow to characterize the properties of the network with regard to the resources flow between players. To the best of our knowledge theses tools have never been used in the network formation literature.

\section{Appendix}

Lemma 1 Suppose the payoff function of each player $i$ satisfies (1) and let $\boldsymbol{g}$ be a non-empty strict Nash network. There exists $i_{0} \in N$ such that $j \succeq_{\boldsymbol{g}} i_{0}$ for all $j \in N$.

Proof Let $\boldsymbol{g}$ be a non-empty strict Nash network. We know that $i \succeq_{\boldsymbol{g}} i$ by reflexivity of $\succeq_{\boldsymbol{g}}$ over $N$. We need to show that there is $i_{0} \in N$ such that $j \succeq_{\boldsymbol{g}} i_{0}$ for all $j \in N \backslash\left\{i_{0}\right\}$. There exists $i_{0}$ such that (i) there is $j \in N: j i_{0} \in A(\boldsymbol{g})$, and (ii) if $i_{0} \succeq_{\boldsymbol{g}} k$, then $k \succeq_{\boldsymbol{g}} i_{0}$ for $k \in N$. (i) comes from the fact that $\boldsymbol{g}$ is non empty and (ii) comes from the fact that $N$ is finite and $\succeq_{\boldsymbol{g}}$ is transitive. We can now introduce a contradiction. Suppose that there is a player $i^{\prime} \in N$ such that $i^{\prime} \nsucceq_{\boldsymbol{g}} i_{0}$. We have $i_{0} \nsucceq_{\boldsymbol{g}} i^{\prime}$ otherwise $i_{0}$ has not been well chosen. Since there is a player $j$ such that $j i_{0} \in A(\boldsymbol{g})$ and $\boldsymbol{g}$ is a strict Nash network we have $\pi_{j}\left(A_{j}(\boldsymbol{g}), A_{-j}(\boldsymbol{g})\right)-\pi_{j}\left(A_{j}(\boldsymbol{g})-j i_{0}, A_{-j}(\boldsymbol{g})\right)>0$. By the partner heterogeneity assumption on value and cost and the assumption $i^{\prime} \nsucceq_{\boldsymbol{g}} i_{0}$ and since $N_{i^{\prime}}(A(\boldsymbol{g})) \cap N_{i}(A(\boldsymbol{g}))=\emptyset$, we have $\pi_{i^{\prime}}\left(A_{i^{\prime}}(\boldsymbol{g})+i^{\prime} i_{0}, A_{-i^{\prime}}(\boldsymbol{g})\right)-\pi_{i^{\prime}}\left(A_{i^{\prime}}(\boldsymbol{g}), A_{-i^{\prime}}(\boldsymbol{g})\right) \geq \pi_{j}\left(A_{j}(\boldsymbol{g}), A_{-j}(\boldsymbol{g})\right)-\pi_{j}\left(A_{j}(\boldsymbol{g})-j i_{0}, A_{-j}(\boldsymbol{g})\right)>$ 0. It follows that $\boldsymbol{g}$ is not a strict Nash network, a contradiction. 
Lemma 2 Suppose the payoff function of each player $i$ satisfies (1) and let $\boldsymbol{g}$ be a non-empty strict Nash network. Then there does not exist four players $i, j, k, \ell$ in $N$ such that $i \ell, j \ell, k j \in A(\boldsymbol{g})$ and player $i$ and $j$ do not form other arcs in $\boldsymbol{g}$.

Proof Let $\boldsymbol{g}$ be a non-empty strict Nash network. To introduce a contradiction suppose four

players $i, j, k, \ell$ in $N$ such that $i \ell, j \ell, k j \in A(\boldsymbol{g})$. By BPSN player $k$ has formed no arc with player $\ell$ and since $\boldsymbol{g}$ is strict Nash, we have:

$$
\pi_{k}\left(A_{k}(\boldsymbol{g}), A_{-k}(\boldsymbol{g})\right)-\pi_{k}\left(A_{k}(\boldsymbol{g})+k i-k j, A_{-k}(\boldsymbol{g})\right)=c_{j}-c_{\ell}-v_{j}<0
$$

Similarly, player $i$ has formed an arc with player $\ell$ in $\boldsymbol{g}$, so by BPSN, $i$ has not formed an arc with $j$ in $\boldsymbol{g}$. Since $\boldsymbol{g}$ is a strict Nash network, we have:

$$
\pi_{i}\left(A_{i}(\boldsymbol{g}), A_{-i}(\boldsymbol{g})\right)-\pi_{i}\left(A_{i}(\boldsymbol{g})+i j-i \ell, A_{-i}(\boldsymbol{g})\right)=c_{\ell}-c_{\jmath}+v_{j}<0
$$

Equations 2 and 8 cannot be satisfied simultaneously, a contradiction.

\section{References}

[1] Venkatesh Bala and Sanjeev Goyal. A noncooperative model of network formation. Econometrica, 68(5):1181-1230, September 2000.

[2] M. E. J. Newman. The Structure and Function of Complex Networks. SIAM Review, 45(2):167$256,2003$.

[3] Matthew O. Jackson. Social and Economic Networks. Princeton University Press, August 2008.

[4] Robert P. Gilles and Cathleen Johnson. Spatial social networks. Review of Economic Design, $5(3): 273-299,2000$.

[5] Michael McBride. Position-specific information in social networks: Are you connected? Mathematical Social Sciences, 56(2):283-295, September 2008. 
[6] Hans Haller and Sudipta Sarangi. Nash networks with heterogeneous links. Mathematical Social Sciences, 50(2):181-201, September 2005.

[7] Daniel A. Hojman and Adam Szeidl. Core and periphery in networks. Journal of Economic Theory, 139(1):295-309, March 2008.

[8] Andrea Galeotti, Sanjeev Goyal, and Jurjen Kamphorst. Network formation with heterogeneous players. Games and Economic Behavior, 54(2):353-372, February 2006.

[9] Pascal Billand, Christophe Bravard, and Sudipta Sarangi. Strict Nash Networks and Partner Heterogeneity. International Journal of Game Theory, 2010, Forthcoming.

[10] Andrea Galeotti. One-way flow networks: the role of heterogeneity. Economic Theory, 29(1):163-179, September 2006.

[11] Frank Harary, Robert Z. Norman, and Dorwin Cartwright. Structural Models: An Introduction to the Theory of Directed Graphs. John Wiley \& Sons Inc, New York, 1965.

[12] Garrett Birkhoff. Lattice theory. volume 25. American Mathematical Society, 1993.

[13] Albert-Lszl Barabsi, Rka Albert, and Hawoong Jeong. Scale-free characteristics of random networks: the topology of the world-wide web. Physica A: Statistical Mechanics and its Applications, 281(1-4):69 - 77, 2000. 